\title{
Metabolomics of gingival crevicular fluid to identify biomarkers for periodontitis: A systematic review with meta-analysis
}

\author{
Giacomo Baima $^{1} \odot$ | Matteo Corana ${ }^{1}$ | Giovanni laderosa ${ }^{1}$ | Federica Romano ${ }^{1}$ [ | \\ Filippo Citterio $^{1}$ (i) | Gaia Meoni ${ }^{2}$ | Leonardo Tenori ${ }^{3,4}$ | Mario Aimetti ${ }^{1}$ (c)
}

\footnotetext{
${ }^{1}$ Department of Surgical Sciences, C.I.R. Dental School, University of Turin, Turin, Italy

${ }^{2}$ Giotto Biotech S.R.L, Sesto Fiorentino, Florence, Italy

${ }^{3}$ Magnetic Resonance Center (CERM), University of Florence, Sesto Fiorentino,

Florence, Italy

${ }^{4}$ Department of Chemistry, University of Florence, Sesto Fiorentino, Florence, Italy

Correspondence

Giacomo Baima, Department of Surgical Sciences, C.I.R. Dental School, University of Turin, Turin, Italy.

Email: giacomo.baima@gmail.com
}

\begin{abstract}
The present systematic review aimed to examine periodontitis-specific biomarkers in the gingival crevicular fluid (GCF) that could have a diagnostic relevance, and to provide a qualitative assessment of the current literature. Metabolites are reliable indicators of pathophysiological statuses, and their quantification in the GCF can provide an outlook of the changes associated with periodontitis and have diagnostic value. Relevant studies identified from PubMed, Embase, Cochrane Library, and Scopus databases were examined to answer the following PECO question: "In systemically healthy individuals, can concentration of specific metabolites in the GCF be used to discriminate subjects with healthy periodontium $(\mathrm{H})$ or gingivitis from patients with periodontitis $(P)$ and which is the diagnostic accuracy?" Quality of included studies was rated using a modified version of the QUADOMICS tool. Meta-analysis was conducted whenever possible. After the screening of 1,554 titles, 15 studies were selected, with sample size ranging from 30 to 93 subjects. Eleven studies performed targeted metabolomics analysis and provided data for 10 metabolites. Among the most consistent markers, malondialdehyde levels were found higher in the $\mathrm{P}$ group compared with $\mathrm{H}$ group (SMD $=2.86 ; 95 \% \mathrm{Cl}$ : 1.64, 4.08). Also, a significant increase of 8-hydroxy-deoxyguanosine, 4-hydroxynonenal, and neopterin was detected in periodontally diseased sites, while glutathione showed an inverse trend. When considering data from untargeted metabolomic analysis in four studies, more than $\mathbf{4 0}$ metabolites were found significantly discriminant, mainly related to amino acids and lipids degradation pathways. Notably, only one study reported measures of diagnostic accuracy. Several metabolites were differentially expressed in GCF of subjects across different periodontal conditions, having a major potential for investigating periodontal pathophysiology and for site-specific diagnosis. Oxidative stress-related molecules, such as malondialdehyde and 8-hydroxy-deoxyguanosine, were the most consistently associated to periodontitis (PROSPERO CRD42020188482).
\end{abstract}

KEYWORDS

biomarkers, gingival crevice fluid, metabolomics, periodontal diseases 


\section{1 | INTRODUCTION}

Periodontitis is a chronic inflammatory disease that causes the irreversible destruction of periodontal tissues and may lead to tooth loss and even negative systemic outcomes if not promptly treated. ${ }^{1-3}$ The disease is underpinned by a multifactorial etiology, encompassing systemic risk indicators, bacterial dysbiosis and host immune dysregulations. ${ }^{4,5}$ Regarding its clinical course, periodontitis is characterized by a nonlinear pattern of progression, with multiple burst of activity alternated to periods of remission. ${ }^{6}$ Key to intercept early stages of activity and to prevent further tissue destruction is to validate an accurate periodontal diagnostic tool. Current clinical and radiographic diagnostic methods do not fulfill these requirements and are more reliable to assess past tissue destruction. ${ }^{7}$ Therefore, molecular biomarkers for disease activity are needed.

In this context, omics sciences have been regarded as powerful tools for biomarker discovery and validation in medicine. ${ }^{8,9}$ Among these, metabolomics deals with the collective characterization and quantification of pools of metabolites in biofluids or tissues employing spectroscopic assay techniques. ${ }^{10,11}$ The metabolome comprises a broad range of endogenous and exogenous compounds and can be affected by both genetic and environmental factors. ${ }^{12,13}$ Since metabolites stand as endpoints of complex pathways driving the initiation and progression of periodontitis, metabolomics can be used in a variety of applications, including early disease detection, assessment of the current disease status/activity, and monitoring of the response to treatment. ${ }^{14-17}$ Furthermore, a detailed examination of the triggered or altered metabolic pathways may deepen scientific knowledge on the complex pathogenic mechanisms of periodontitis, both at microbiological and immunological scale. ${ }^{9}$

To this regard, gingival crevicular fluid (GCF) has attracted the interest of periodontal research because of its non-invasiveness and its potential to mirror periodontal conditions. ${ }^{18,19} \mathrm{GCF}$ is a complex biofluid formed as a blood ultrafiltrate through the periodontal sulcus/pocket cells and thus contains many bacteria- or host-derived metabolites that can be used as a site-specific biomarker of disease activity. ${ }^{20,21}$

In recent years, interest on metabolites as indicators of periodontal status has increasingly grown. At the same time, diagnostic and prognostic accuracy of GCF contents has been assessed by a vast amount of literature, mainly focusing on proteomic or oxidative stress biomarkers. ${ }^{21,22}$ To the best of our knowledge, no systematic review exists on the diagnostic ability for periodontitis of GCF-derived metabolites. Integrating evidence on this topic would help refining the knowledge on pathophysiological mechanisms and identifying new non-invasive biomarkers of disease activity. Therefore, the aims of the present systematic review were to examine periodontitis-specific biomarkers in the GCF that could have a diagnostic relevance, and to provide a qualitative assessment of the current literature.

\section{2 | MATERIAL AND METHODS}

This research has been conducted in accordance with the Cochrane Handbook $^{23}$ and reported according to the PRISMA guidelines. ${ }^{24}$ The protocol was registered at the International Prospective Register of Systematic Reviews (PROSPERO) under the number CRD42020197691.

\subsection{Focused questions}

This systematic review was designed to answer the following focused question:

In systemically healthy individuals, can concentration of specific metabolites in the GCF be used to discriminate subjects with periodontal healthy conditions $(\mathrm{H})$ or gingivitis $(G)$ from patients with periodontitis $(P)$ and which is their diagnostic accuracy?

(P) Population. Adult patients in good systemic health.

(E) Exposure. Patients or sites with a clinical diagnosis of $\mathrm{P}$.

(C) Comparison. Subjects or sites with $\mathrm{H}$ or $\mathrm{G}$ conditions.

(O) Type of outcome measures. The main outcome of the present systematic review was change in GCF metabolite concentration in $\mathrm{P}$ compared to $\mathrm{H}$ or $\mathrm{G}$ groups. The secondary outcome was any measure of diagnostic accuracy (sensitivity, specificity, receiveroperating characteristic (ROC) curve).

\section{2 | Eligibility criteria}

\section{\begin{tabular}{l|l}
2.2 .1 & Types of Studies
\end{tabular}}

Human studies on metabolomic biomarkers in GCF that provided results on diagnostic accuracy in patients with a diagnosis of P. Not expecting a great number of studies reporting diagnostic accuracy, all original comparative studies comprising the analysis of GCF-derived metabolites were included. Both prospective and retrospective designs were accepted.

\subsection{2 | Control and target condition}

The target conditions were chronic (CP) and aggressive (AgP) forms of $\mathrm{P}$, regardless of the severity and extent. As controls, $\mathrm{G}$ and $\mathrm{H}$ conditions were considered. The diagnosis was considered both at patient and site level. Only studies comparing P patients versus $\mathrm{H}$ or $\mathrm{G}$ subjects were selected. 


\subsection{3 | Reference standard}

The diagnosis of periodontitis was based on clinical parameters (CAL or PD) or a combination of clinical and radiographic parameters. Any definition provided by the authors was accepted.

\subsection{4 | Other exclusion criteria}

Studies involving pregnant females or subjects suffering from systemic diseases were excluded. Furthermore, clinical case reports, literature reviews, editorials, animal studies, and in vitro experiments were also excluded.

\subsection{Search methods for the identification of studies}

The search was performed in duplicate by two calibrated reviewers ( $M C$ and $\mathrm{Gl}$ ) on four electronic databases [National Library of Medicine (Medline via PubMed), Embase, Cochrane Library and Scopus] until October 2020 without any restriction on date of publication or publication status. Combinations of controlled terms (MeSH and EMTREE) and keywords were used as follows:

("metabolomics" OR "metabonomics" OR "metabolites" OR "mass spectrometry" OR "nuclear magnetic resonance spectroscopy") AND ("periodontitis" OR "periodontal disease" OR "gingivitis" OR "oral health") AND ("fluid, gingival crevicular" OR "gingival crevicular").

In addition, the references of all included studies and previous systematic reviews were cross-checked by two reviewers (MC and $\mathrm{GI}$ ) and an additional search on relevant periodontal journals (Journal of Dental Research, Journal of Clinical Periodontology, Journal of Periodontal Research and Journal of Periodontology) was performed from 2010 to 2020 to ensure complete data collection.

\subsection{Study selection}

The titles and abstracts of potentially eligible studies were screened independently by two reviewers (MC and GI). Studies that met the inclusion criteria, or for which there was insufficient data were selected for full review and assessed independently by two reviewers (GB and GI). Any disagreement was resolved by discussion or by consultation with a third reviewer (FC) when necessary. The reasons for exclusion of studies after full-text analysis were recorded. The inter-reviewer reliability (kappa coefficient) of the screening method was calculated.

\subsection{Data extraction and management}

Data were independently extracted by two authors (MC and $\mathrm{Gl}$ ) using specifically designed data extraction forms. Any disagreement was resolved by discussion with a third reviewer (GB). In the case of prospective intervention studies on $\mathrm{P}$, only data recorded before the periodontal treatment (baseline) were extracted and analyzed. Similarly, in the case of studies comparing periodontal status in patients with and without specific medical conditions (ie, diabetes, obesity), only parameters of systemically healthy individuals were entered in this systematic review.

For each study, the following data were recorded: general characteristics of the study (first author, year of publication, and country/region of origin); study design; characteristics of the population (number of patients, inclusion/exclusion criteria, age, smoking habits, definition of $\mathrm{P}$ and $\mathrm{G}$ or $\mathrm{H}$ conditions, periodontal parameters); outcome assessment [GCF collection protocol (presampling procedures, hour of collection, sites, collection method, time, measurement of volume), pre-analytical procedures (sampling storage method, sample preparation), detection methods]; and main findings (type and concentration of metabolites identified in $\mathrm{P}$ and control groups, unit of measure, measures of diagnostic accuracy).

\subsection{Risk of bias in the included studies and quality assessment}

The risk of bias and quality assessment of the included studies was completed independently by two reviewers (MC and $\mathrm{Gl}$ ) as part of the data extraction process using a modified version of the NIH Quality Assessment Tool for Observational Cohort and CrossSectional Studies and the QUADOMICS tool. This later, developed to evaluate quality issues specific to omics research, comprises of 15 items analyzing research question, study population, exposure, GCF sampling procedures (including use of paper strips, duration of sampling, volume determination), confounding factors (smoking habits and systemic diseases affecting GCF metabolic profile, ie, diabetes mellitus), outcomes, and statistical analysis. Every item was given either 0 or 1 point, apart from item 8,12, and 14 on sampling procedures and confounders in which each aspect accounted for 0.25 points. The final scores are interpreted as follows: $0-4$, very low quality; 5-8, low quality; 9-12, moderate quality; and 13-16, high quality.

\section{7 | Strategy for data synthesis}

For targeted analysis, the level of the metabolites was reported as mean \pm standard deviation (SD), median (interquartile range, IQR), or median (min-max). When at least two studies reported mean concentration of the same biomarker, standardized weighted mean differences (SMD) and 95\% confidence intervals (95\% Cl) were calculated using a random effect model. ${ }^{25}$ The statistical heterogeneity among studies was assessed using the $\mathrm{Q}$ test as well as the $\mathrm{I}^{2}$ index. A, percentage of $\mathrm{I}^{2}$ from $25 \%$ to $50 \%$ was considered as low heterogeneity, $50 \%$ to $75 \%$ as medium heterogeneity, and $>75 \%$ as high heterogeneity. Forest plots were created to illustrate the effects of 
the different studies and the global estimation of the meta-analysis. Statistical significance was defined by a $p$ value $\leq .05$.

For untargeted studies, only significantly discriminant metabolites between cases and controls were tabulated.

\section{RESULTS}

\section{1 | Study selection}

In total, 2,102 articles were identified by the electronic search and 6 additional articles by manual search. After the removal of duplicates, 1,554 titles and abstracts were screened for eligibility and 24 papers were analyzed in full text. Of these, 9 articles were excluded for different reasons (Supplementary Table S1), and 15 papers were selected for the qualitative analysis. The measure of inter-reviewer agreement was $k=0.91$ for abstract screening and $k=0.97$ for fulltext analysis. The study flowchart is depicted in Supplementary Figure S1.

\section{2 | Study characteristics}

Supplementary Table S2 summarizes the general information about the included studies. All the identified entries were published between 2007 and 2020. Six studies were conducted in Turkey, 3 in China, 2 in Japan, and the others were from USA, Egypt, India, and Sudan. The total sample size ranged from 30 to 93, with cases ranging from 15 to 41 and controls ranging from 14 to 46 . Most of the studies presented a cross-sectional design. Four studies performed untargeted metabolomics, ${ }^{26-29}$ while the remaining 11 studies aimed at identifying targeted metabolites within the GCF. ${ }^{30-40}$

Periodontitis case definitions and periodontal parameters were quite heterogeneous across the studies. Twelve articles enrolled patients with CP, while 4 articles patients with AgP. Only 3 studies included $\mathrm{G}$ as control group instead of $\mathrm{H}$.

Details for GCF collection and analytical protocols are summarized in Table 1. Only 8 studies described the pre-sampling procedures, ${ }^{31,34-36,38-40}$ while five studies detailed the hour of sample collection. ${ }^{31,33,34,38,40}$ The majority of studies used paper strips (Periopaper) to collect GCF; while few studies used endodontic paper points ${ }^{37}$ and microcapillary pipettes. ${ }^{39}$ Eleven studies reported a sampling duration of $30 \mathrm{~s} ;{ }^{26,27,29,31-36,38,40} 2$ studies of $10 \mathrm{sec}-$ onds; ${ }^{28,30}$ while 2 other studies did not mention it. ${ }^{37,39}$ Nine studies used Periotron 8000 for volume measurement. ${ }^{26,29-32,35,36,38,40}$ Mass spectrometry (MS), either coupled with liquid or gas chromatography (LC or GC), was the preferred method for untargeted analysis; while for targeted analysis the detection methods were strictly dependent on the metabolite tested as summarized in the last column of Table 1.

Table 2 illustrates in details the results from included studies. A total of 10 metabolites were analyzed across the 11 studies adopting a targeted metabolomic analysis; while more than 300 different
GCF analytes were recognized in studies performing untargeted metabolomics analysis. Malondialdehyde (MDA) was the most investigated molecule, recurring in four studies ${ }^{31,32,38,40}$ followed by 8-hydroxy-deoxyguanosine (8-OHdG) in three studies, ${ }^{33,35,36}$ and lysophosphatidic acid (LPA) in two studies. ${ }^{30,37}$ LPA precursors, lysophosphatidylcholine (LPC), and lysophosphatidylethanolamine (LPE), together with choline, 4-hydroxynonenal (4-HNE), neopterin, and reduced and oxidized glutathione were only evaluated in one article. Analysis of diagnostic accuracy was performed in only one study. ${ }^{29}$ ROC analysis displayed that the panel of citramalic acid and $\mathrm{N}$-carbamylglutamate provided satisfactory accuracy for the diagnosis of generalized CP (area under the curve $=0.876$ ).

\section{3 | Synthesis of results}

\subsubsection{Findings from specific metabolites}

Meta-analysis was conducted only for MDA, for which data were available in 4 studies involving 255 participants (Figure 1). Patients with $\mathrm{P}$ showed higher levels of MDA compared with $\mathrm{H}$ subjects $(\mathrm{SMD}=2.86 ; 95 \% \mathrm{Cl}: 1.64,4.08)$ with high heterogeneity $(p<.001$, $\left.I^{2}=89.6 \%\right)$.

8-OHdG was also investigated across three different studies ${ }^{33,35,36}$ and revealed a consistent tendency to gradually increase from $\mathrm{H}$ to $\mathrm{G}$ subjects, and among $\mathrm{P}$ patients from healthy to diseased sites. Unfortunately, heterogeneity in reporting the outcome measures did not allow a meta-analysis of the results, and authors were not available for providing them.

For LPA, conflicting results were obtained. In particular, one study ${ }^{37}$ reported a marked increase in $\mathrm{CP}$ and $\mathrm{AgP}$ groups compared with $\mathrm{H}$ controls for all the four tested LPA species; whereas another study ${ }^{30}$ obtained significantly higher LPA concentrations in $\mathrm{H}$ subjects, although numerical values were not given.

For the remaining markers, only isolated reports were available. ${ }^{30}$ Among LPA precursors, LPC increased in the P group compared with $\mathrm{H}$ controls, together with choline; whereas LPE followed the opposite trend. 4-HNE level in GCF of $\mathrm{P}$ patients was found higher than $\mathrm{H}$ participants in one study ${ }^{35}$ although statistical significance was reached solely among smokers. Neopterin levels were measured across four different clinical periodontal conditions, with significant higher concentrations encountered within the AgP group compared with the others. Finally, reduced and oxidized glutathione was quantified by one study, ${ }^{33}$ which attested a marked decrease of both molecules within CP group.

\subsection{2 | Untargeted analysis}

In a recent study, ${ }^{29} 147$ different analytes were obtained after untargeted profiling of 174 GCF samples. The most significantly enriched metabolites in $\mathrm{CP}$ group, uracil, $\mathrm{N}$-carbamylglutamate $2, \mathrm{~N}$ - acetyl$\beta$-D-mannosamine 1 , fructose 1 , citramalic acid, 5- dihydrocortisol 
3, and 4-hydroxyphenylacetic acid were also positively correlated to more severe clinical parameters, while the opposite trends were observed for thymidine 3 and O-phosphoserine 1 .

In one study, ${ }^{26}$ a total of 349 different metabolites were qualitatively assesses in the GCF. Noradrenaline, uridine, $\alpha$-tocopherol, dehydroascorbic acid, xanthine, galactose, glucose-1-phosphate, and ribulose-5-phosphate levels were among the most significantly increased metabolites in patients with GAgP, while thymidine, glutathione, and ribose-5-phosphate levels were decreased.

Another study identified 19 metabolites using GC-MS. ${ }^{27}$ Putrescine, lysine, phenylalanine, ribose, taurine, 5-aminovaleric acid, and galactose displayed a trend toward increasing in deep pocket sites; whereas lactic acid, benzoic acid, glycine, malic acid, and phosphate showed a gradual decrease from severe periodontitis sites to healthy sites.

A precedent article characterized a number of 51 pro-resolving and pro-inflammatory lipid mediators in the GCF. ${ }^{28}$ Ratios of $\omega 3$ - to $\omega$ 6-polyunsatured fatty acids (PUFAs) and of the direct precursors of the pro-resolution lipid mediators were significantly lower in the GCF of AgP patients than in the healthy controls.

\subsection{Quality assessment}

Supplementary Table S3 reports the results of the quality assessment using the QUADOMICS tool. While the almost totality of the studies adopted rational and replicable criteria for $\mathrm{P}, \mathrm{G}$, and $\mathrm{H}$ subjects selection (Items 2-7), operative protocol and reporting were less rigorous for GCF sampling and statistical analysis. According to the present criteria, only one study was considered as high quality, ${ }^{39}$ nine studies as moderate quality, ${ }^{26,29,31-36,38,40}$ while the others were rated as low quality. ${ }^{27,28,30,37}$

\section{4 | DISCUSSION}

GCF is considered the most proximal biofluid to investigate periodontal disease pathophysiology, while metabolites are the most proximal reporters of pathophysiological states. ${ }^{9,18}$ The present study was the first systematic review to assess the relationship between GCF derived metabolites and periodontal disease status. Several molecules were investigated across the 15 included reports, with consistent data available for some of them. Only one untargeted study reported measures of diagnostic accuracy; thus, the qualitative and quantitative syntheses of the results have been restricted to mean concentrations of target metabolites in GCF of both $\mathrm{P}$ patients and $\mathrm{H}$ controls.

\section{1 | Results for specific metabolites}

Targeted metabolomic analyses are indicated when specific hypothesis-driven metabolites are sought. Oxidative stress-related metabolites were the most investigated in targeted GCF analyses. This tendency is based on the rationale that oxidative stress is one of the key pathophysiological mechanisms underpinning periodontitis at the molecular scale. ${ }^{41} \mathrm{~A}$ systematic review previously published ${ }^{22}$ specifically focused on oxidative stress-related markers and some of the current results coincide. Among these, MDA derives from the peroxidation of PUFA, and it is considered a reliable indicator of reactive oxygen species (ROS) induced cellular damage. ${ }^{22,42}$ From the present meta-analysis, a markedly higher GCF level of MDA was found in $\mathrm{P}$ patients compared with $\mathrm{H}$ controls, indicating an increased inflammatory mediated oxidative stress within the periodontal microenvironment. In fact, ROS released from neutrophils after interactions with pathogenic antigens directly destroy cell membrane and initiate the pathway of lipid peroxidation, which in turn elicits more inflammation and tissue degradation. ${ }^{43}$

Also, 8-HOdG is a stable marker of oxidative DNA damage, and it is another steady indicator for the harmful effects of ROS. Its increase in biofluids has been demonstrated across different medical conditions ${ }^{44,45}$ among which periodontitis. ${ }^{46}$ Three included studies reported consistent upregulated concentrations of 8-HOdG within $\mathrm{P}$ individuals, although meta-analysis of results was not achievable. The evidence that 8-HOdG can serve as a marker of active disease is supported by its tendency to gradually increase across different periodontal conditions, including $\mathrm{H}, \mathrm{G}$, and moderate to severe P. In addition, some trials demonstrated a significant reduction of this molecule after periodontal therapy. ${ }^{35,36}$

Among other oxidative stress-related metabolites, 4-HNE is a major aldehydic end product also derived from lipid peroxidation processes, which possesses strong cytotoxic and signaling activities. ${ }^{47}$ As for above, its concentrations have been reported significantly overexpressed in GCF of P group, with a marked tendency to increase among smoking patients. ${ }^{35}$

LPAs are a group of phospholipid mediators that plays a key role in inflammation and their contribution in periodontitis pathogenesis is supported by several lines of evidence. ${ }^{37,48}$ Because of the differences in the responses produced by various LPAs, it is important to detect individual LPA species rather than total LPAs. Unexpectedly, values of LPA species in this review conflicted among two studies. Bathena et $\mathrm{al}^{37}$ were the first to develop a method for LPA quantification in saliva and GCF, encountering significantly higher levels for 4 LPA species (18:0, 16:0, 18:1, and 20:4) in P compared with $\mathrm{H}$ sites. On the other hand, Hashimura et $\mathrm{al}^{30}$ detected a lower concentration of LPA in GCF of P patients, that plausibly attributed to higher lysophospholipase activity. In the same report, concentrations of LPA precursors LPE and LPC were also quantified, although their differences between $\mathrm{P}$ and $\mathrm{H}$ were rather moderate. Unfortunately, the two studies were quite deficient in reporting populations and methods, and further evidence is needed to thoroughly address the issue.

Conversely, reduced glutathione (GSH) is a crucial intracellular antioxidant for ROS detoxification and may serve as an indicator of the antioxidant defense status. ${ }^{49}$ GSH levels in the GCF have been found significantly decreased within the P group. ${ }^{33}$ Interestingly, the same study revealed that obesity in patients with $\mathrm{CP}$ is associated 
TABLE 1 Methods of GCF collection and sample analysis across the included studies

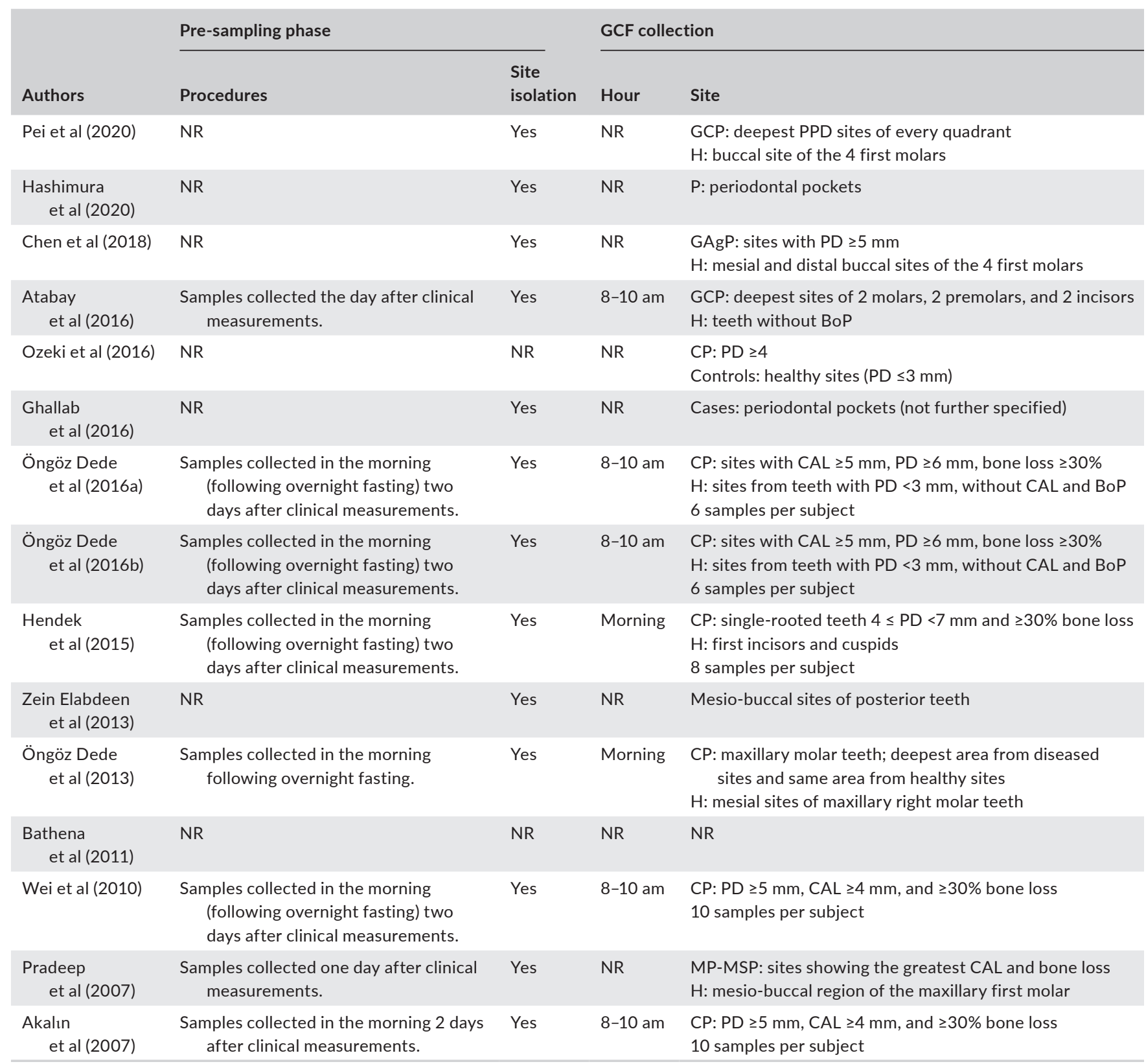

Abbreviations: BoP, bleeding on probing; CAL, clinical attachment level; CP, chronic periodontitis; ELISA, enzyme-linked immunosorbent assay; FFA, free fatty acids; GAgP, generalized aggressive periodontitis; GC-MS, gas chromatography-mass spectrometry; GCP, generalized chronic periodontitis; GC-TOF-MS, gas chromatography time-of-flight mass spectrometry; $\mathrm{H}$, periodontally healthy individuals; HPLC, high performance liquid chromatography; LC-MS/MS, liquid chromatography-tandem mass spectrometry; LPA, lysophosphatidic acid; LPC, lysophosphatidylcholine; LPE, lysophosphatidylethanoamine; MP, mild periodontitis; MSP, moderate to severe periodontitis; NBT, nitroblue tetrazolium; NR, not reported; P, periodontitis patients; PD, pocket depth.

with even decreased GSH levels and that nonsurgical periodontal treatment is able to reconstitute higher values.

\section{2 | Untargeted GCF metabolomics}

By using an untargeted omic approach, there is the advantage of scaling up from a single- to a multi-parameter model, getting an extensive and non-hypothesis driven view of the shifts within the metabolic profiling of $\mathrm{P}$ and $\mathrm{H}$ controls., ${ }^{9,50}$ Comprehensively, the results of the present review support the conclusion of a distinctive metabolomic signature of periodontitis in GCF. Metabolites are considered the most proximal reporters of physiopathological states at the histological level, more than proteomic or transcriptomic variations. ${ }^{51}$ The metabolic changes encountered implicate pathways involved in periodontal disease progression and in macromolecular degradation, such as D-glutamine and D-glutamate metabolism, histidine metabolism, and tyrosine metabolism. ${ }^{26,29}$ The overexpression 


\begin{tabular}{|c|c|c|c|c|c|}
\hline \multirow[b]{2}{*}{ Method } & \multirow[b]{2}{*}{ Duration } & \multirow[b]{2}{*}{ Volume } & \multicolumn{2}{|c|}{ Pre-analytical procedures } & \multirow[b]{2}{*}{ Detection method } \\
\hline & & & Sample storage & $\begin{array}{l}\text { Sample } \\
\text { preparation }\end{array}$ & \\
\hline Periopapers & $30 \mathrm{~s}$ & Periotron 8000 & $-80^{\circ} \mathrm{C}$ & Yes & GC-TOF-MS \\
\hline Periopapers & $10 \mathrm{~s}$ & Periotron 8000 & NR & Yes & LC-MS/MS for LPAs; ELISA for choline \\
\hline Periopapers & $30 \mathrm{~s}$ & Periotron 8000 & $-80^{\circ} \mathrm{C}$ & Yes & GC-MS \\
\hline Periopapers & $30 s$ & Periotron 8000 & $-80^{\circ} \mathrm{C}$ & Yes & ELISA \\
\hline Periopapers & $30 \mathrm{~s}$ & NR & $-80^{\circ} \mathrm{C}$ & Yes & GC-MS \\
\hline Periopapers & $30 \mathrm{~s}$ & Periotron 8000 & $-20^{\circ} \mathrm{C}$ & Yes & HPLC \\
\hline Periopapers & $30 \mathrm{~s}$ & NR & $-40^{\circ} \mathrm{C}$ & Yes & Enzyme recycling method \\
\hline Periopapers & $30 \mathrm{~s}$ & NR & $-40^{\circ} \mathrm{C}$ & Yes & $\begin{array}{l}\text { DNA/RNA Oxidation Damage Enzyme } \\
\text { Immunoassay }\end{array}$ \\
\hline Periopapers & $30 \mathrm{~s}$ & Periotron 8000 & $-80^{\circ} \mathrm{C}$ & Yes & ELISA \\
\hline Periopapers & $10 \mathrm{~s}$ & NR & Frozen & Yes & $\begin{array}{l}\text { LC-MS/MS with electrospray ionization } \\
\text { for FFA and eicosanoids }\end{array}$ \\
\hline Periopapers & $30 \mathrm{~s}$ & Periotron 8000 & $-80^{\circ} \mathrm{C}$ & Yes & Enzyme Immunoassay \\
\hline $\begin{array}{l}\text { Endodontic paper } \\
\text { points }\end{array}$ & NR & Digital meter & $-80^{\circ} \mathrm{C}$ & Yes & LC-MS/MS \\
\hline Periopapers & $30 \mathrm{~s}$ & Periotron 8000 & Frozen & Yes & HPLC \\
\hline Microcapillary pipettes & NR & $3 \mu l$ & $-70^{\circ} \mathrm{C}$ & Yes & Enzyme immunoassay \\
\hline Periopapers & $30 \mathrm{~s}$ & Periotron 8000 & PBS (pH 6.5) & Yes & HPLC \\
\hline
\end{tabular}

of glycosidase, lipase, and protease activities is associated with periodontal inflammation and provides a more favorable energetic environment for pathogenic bacteria, exacerbating the disease state. ${ }^{52}$

Metabolites and pathways detected in the GCF present some similarities and some differences from the ones encountered in saliva. ${ }^{53}$ Some factors may be responsible for this separation, primarily the fact that saliva collects components from many other sources than GCF and it is reflective of both the individual systemic status $^{54}$ and the whole mouth infectious-inflammatory status. ${ }^{19}$ Also, GCF characteristics are more strongly influenced by quality of the subgingival ecological niche. ${ }^{29}$ Molecules derived from sugar metabolism, such as fructose, ribose, and galactose, are employed as a source of energy and carbon by oral bacteria and were found consistently elevated within the GCF of P groups. ${ }^{55}$

Finally, the type of analytical platform employed could significantly affect the results, with MS and, especially, nuclear magnetic resonance being the most prevalent for untargeted metabolomics. ${ }^{56,57}$ All the 4 studies included in this review employed LC- or GCMS, providing some discriminant metabolites consistent with those found altered in salivary samples of $P$ patients, such as putrescine, 
잉요

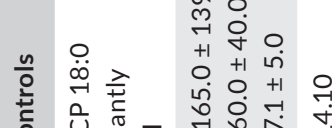

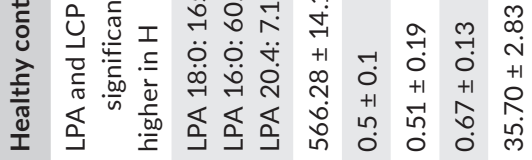

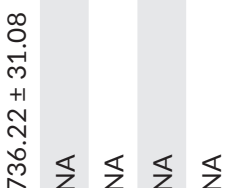

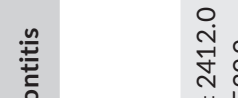

$\stackrel{ }{\circ}$

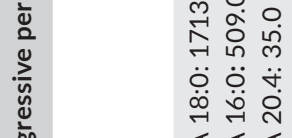

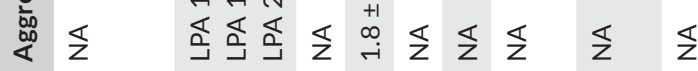

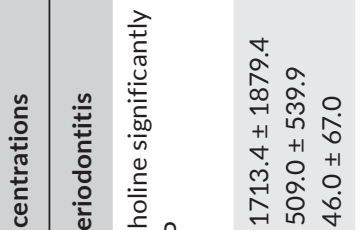

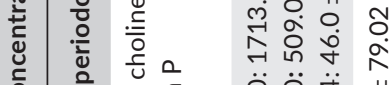

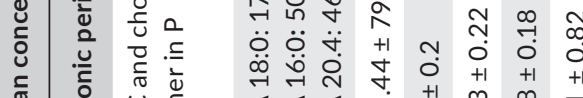

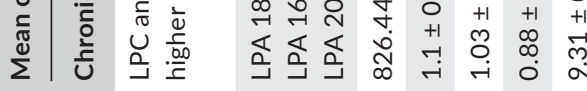

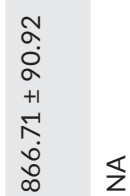

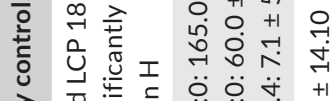

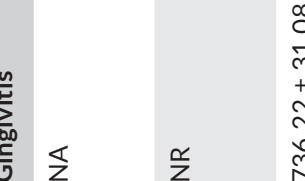

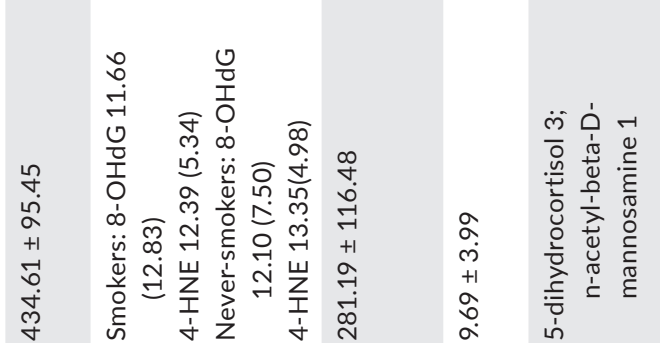

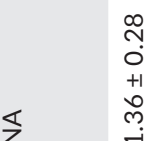

$\stackrel{\infty}{\sim}$
0
+1
+1
$\stackrel{\sim}{\sim}$
$i$

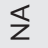

속 ㅇำ

$+1 \frac{\pi}{4}$

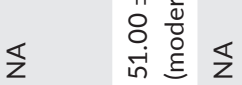
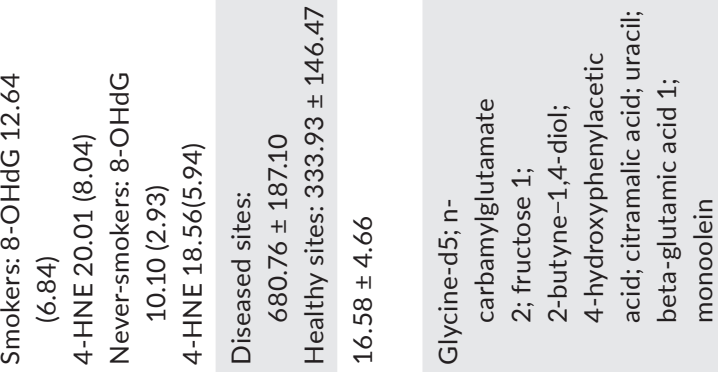

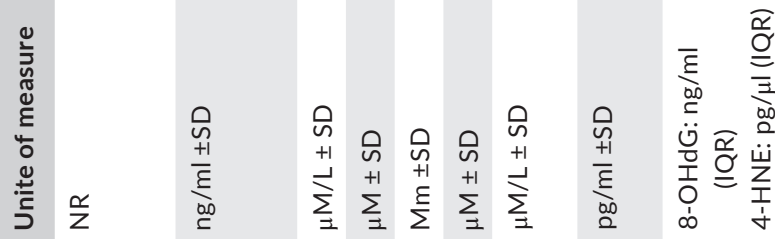

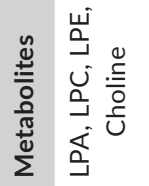

ฐ

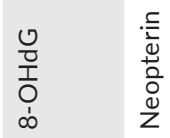

f

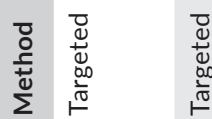

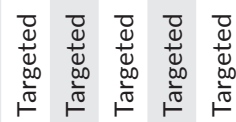

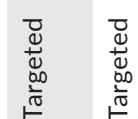

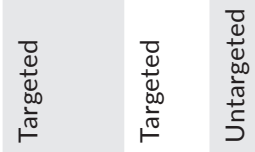

ริํำ

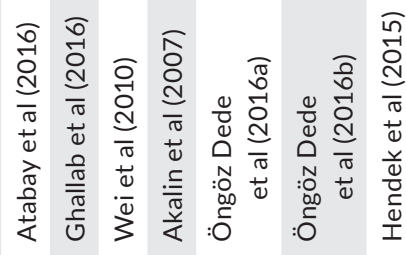

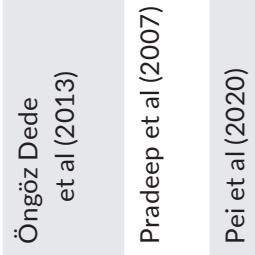




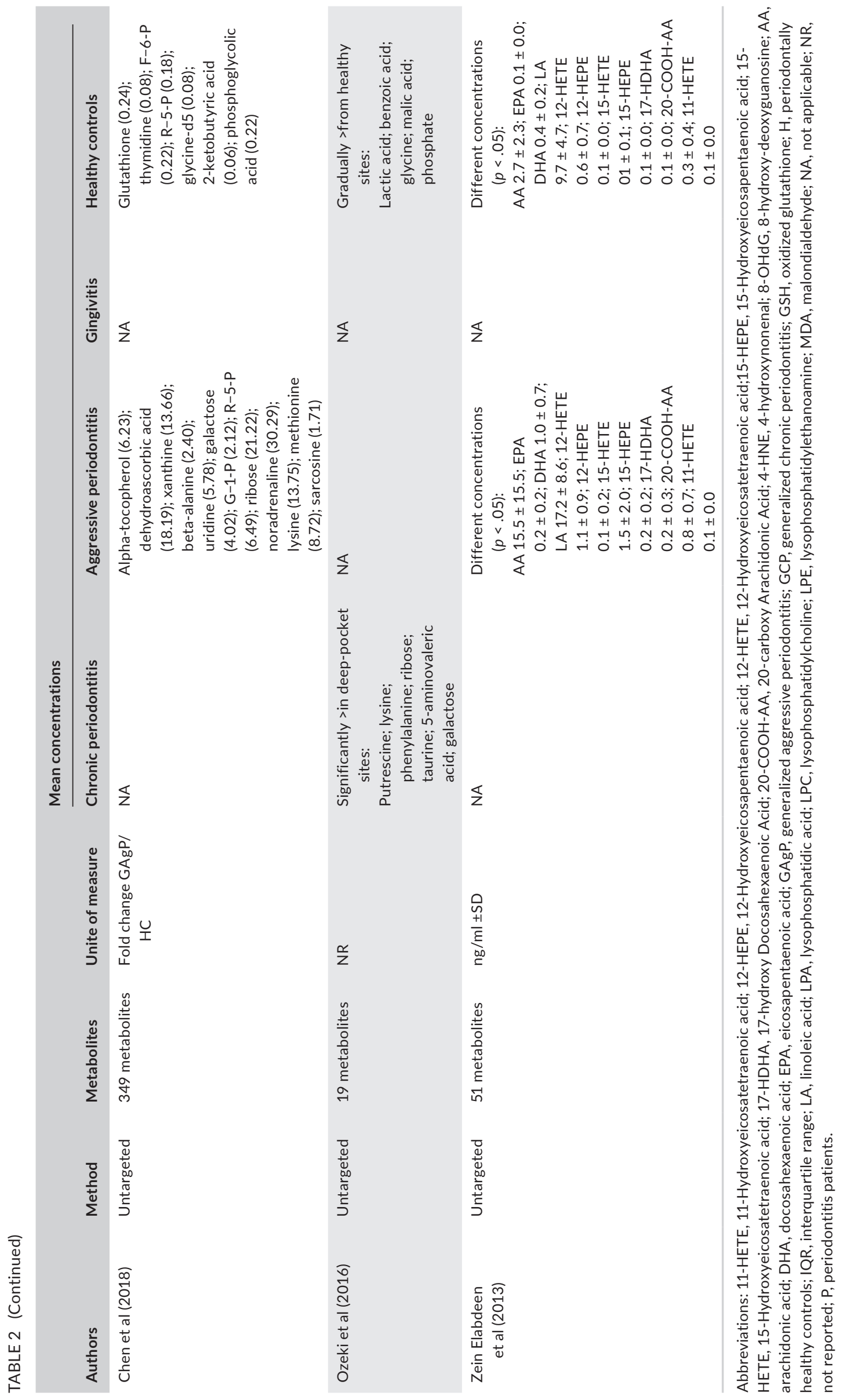




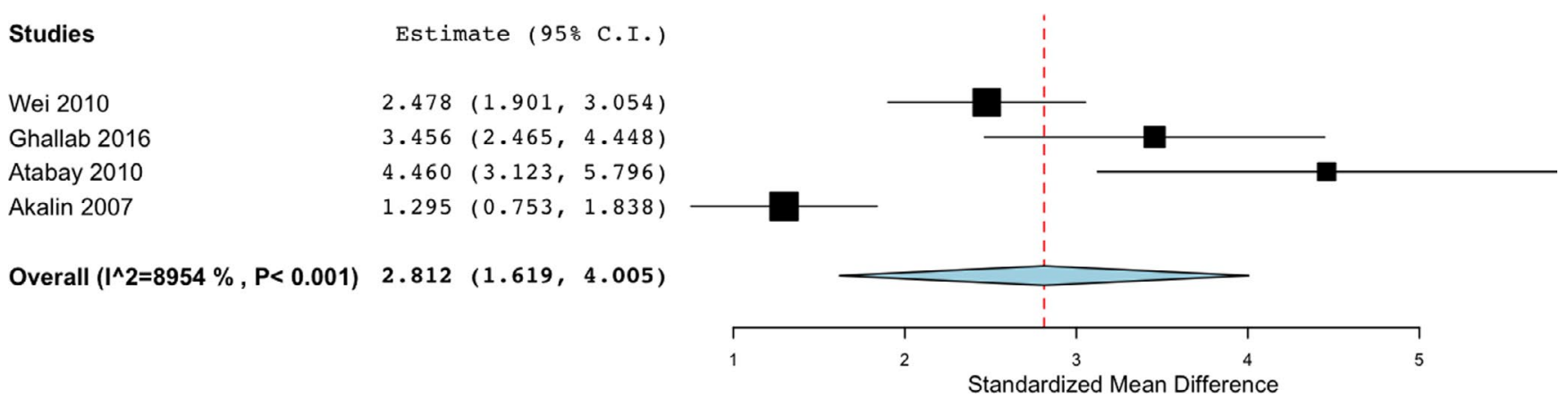

FIGURE 1 Forest plot of meta-analysis of GCF concentration of MDA in periodontitis and condition of periodontal health

lysine, phenylalanine, fructose, galactose, and lactate, the later in creasing in $\mathrm{H}$ controls. ${ }^{15,58}$ Studies concomitantly evaluating metabolites from GCF and saliva are required to properly understand the reciprocal influence of oral biofluids in diagnostic applications.

\section{3 | Limitations and future research directions}

The current work represents the first attempt to systematically summarize the available evidence regarding the utility of GCF metabolites for the diagnosis of periodontitis. Several molecules are differentially expressed within the GCF of subjects with different periodontal conditions, suggesting their potential role as biomarkers. Nevertheless, the data should be interpreted with caution, especially concerning metabolites which failed to reach replication. Moreover, most studies scored moderately low values in the quality assessment evaluation. Limitations comprise the small sample size of the included trials, the heterogenous case definitions employed, together with the lack of standardization of protocols for GCF sampling and processing. In order to allow reproducibility and to improve quality in GCF studies, it is important that sites and methods of GCF collection are transparently described. The diverseness of methods-such as the duration and method of sampling, the storage, and the analytical platform analysis-may introduce confounding in the interpretation of the results and may affect their comparability and generalizability, as already demonstrated for metabolomics studies involving serum and urine. ${ }^{59-61}$

When dealing with GCF analysis, bias may also be introduced by heterogenous criteria for patient and site selection. Severity and activity of periodontal disease are two paramount variables which can influence the GCF biochemistry. ${ }^{62,63}$ A general tendency toward not properly reporting the clinical parameters associated to the sites of collection was observed. Furthermore, only few studies offered a comparative evaluation of $\mathrm{H}$ subjects, and of both diseases and healthy sites in $\mathrm{P}$ patients. This information would be of great value in understanding the biochemical details underpinning activity and quiescence of the disease. Designing longitudinal studies to follow up which $\mathrm{G}$ or $\mathrm{P}$ sites encounter further progression would be valuable, as well as following healing sites after periodontal rigeneration. ${ }^{64,65}$ Proteome quantification and cytokine multiplex assays have already provided strong markers for $\mathrm{P}$, although they are able to detect pathological changes only once they have become clinically evident. ${ }^{18}$ To this regard, the high-resolution information provided by GCF derived metabolites may represent a powerful tool to identify early signs of homeostasis disruption within the periodontal microenvironment.

Overall, this review supports the existence of a metabolomic signature of $\mathrm{P}$ in GCF, together with a potential for metabolic biomarkers in the diagnosis and in the monitoring of disease activity. Consistently with the conclusions of Arias-Bujanda et $\mathrm{al}^{21}$, the present findings corroborated the evidence of an increased scientific attention about the initial discovery of GCF biomarkers. At the same time, a lack of interest in confirming preliminary accuracy results by giving measures of diagnostic accuracy was remarked. These subsequent steps are necessary for a biomarker to be considered clinically reliable. Larger comparative studies and follow-up trials are required to add consistency to these results and pave the way for reliable non-invasive chairside diagnostic tools based on GCF derived metabolites to complement the clinical examination.

\section{CONFLICTS OF INTEREST}

The authors declare that they have no competing interests.

\section{CONSENT TO PARTICIPATE}

Not applicable.

\section{CONSENT FOR PUBLICATION}

Not applicable.

\section{DATA AVAILABILITY STATEMENT}

All data generated or analyzed during this study are included in this published article [and its supplementary information files].

\section{ORCID}

Giacomo Baima (D) https://orcid.org/0000-0002-9395-4967

Federica Romano (D) https://orcid.org/0000-0002-5172-299X

Filippo Citterio (D) https://orcid.org/0000-0002-4513-7479

Mario Aimetti (D) https://orcid.org/0000-0003-0657-0787 


\section{REFERENCES}

1. Tonetti MS, Greenwell H, Kornman KS. Staging and grading of periodontitis: Framework and proposal of a new classification and case definition. J Clin Periodontol. 2018;45:S149-S161. https://doi. org/10.1111/jcpe.12945

2. Romandini M, Baima G, Antonoglou G, Bueno J, Figuero E, Sanz M. Periodontitis, edentulism, and risk of mortality: a systematic review with meta-analyses. J Dent Res. 2021;100(1):37-49. https:// doi.org/10.1177/0022034520952401

3. Romandini M, Laforí A, Romandini P, Baima G, Cordaro M. Periodontitis and platelet count: A new potential link with cardiovascular and other systemic inflammatory diseases. J Clin Periodontol. 2018;45(11):1299-1310. https://doi.org/10.1111/ jcpe.13004

4. Lamont RJ, Koo H, Hajishengallis G. The oral microbiota: dynamic communities and host interactions. Nat Rev Microbiol. 2018;16(12):745-759. https://doi.org/10.1038/s41579-018-0089-x

5. Curtis MA, Diaz PI, Van Dyke TE. The role of the microbiota in periodontal disease. Periodontol 2000. 2020;83(1):14-25. https://doi. org/10.1111/prd.12296

6. Loos BG, Van Dyke TE. The role of inflammation and genetics in periodontal disease. Periodontol 2000. 2020;83(1):26-39. https:// doi.org/10.1111/prd.12297

7. Korte DL, Kinney J. Personalized medicine: an update of salivary biomarkers for periodontal diseases. Periodontol 2000. 2016;70(1):26-37. https://doi.org/10.1111/prd.12103

8. Zhang L, Chen M. An application of ontology-based filtering method in discovery of saliva biomarkers for gastric cancer. In: Proceedings - 2014 IEEE Workshop on Electronics, Computer and Applications, IWECA 2014. 2014:852-855. https://doi.org/10.1109/ IWECA.2014.6845755

9. Nguyen T, Sedghi L, Ganther S, Malone E, Kamarajan P, Kapila YL. Host-microbe interactions: Profiles in the transcriptome, the proteome, and the metabolome. Periodontol 2000. 2020;82(1):115128. https://doi.org/10.1111/prd.12316

10. Takis PG, Ghini V, Tenori L, Turano P, Luchinat C. Uniqueness of the NMR approach to metabolomics. TrAC Trends Anal Chem. 2019;120:115300. https://doi.org/10.1016/j.trac.2018.10.036

11. Vignoli A, Ghini V, Meoni G, et al. High-Throughput Metabolomics by 1D NMR. Angew Chem - Int Ed. 2019;58(4):968-994. https://doi. org/10.1002/anie.201804736

12. Nicholson JK, Holmes E, Kinross JM, Darzi AW, Takats Z, Lindon JC. Metabolic phenotyping in clinical and surgical environments. Nature. 2012;491(7424):384-392. https://doi.org/10.1038/natur e11708

13. Patti GJ, Tautenhahn R, Siuzdak G. Meta-analysis of untargeted metabolomic data from multiple profiling experiments. Nat Protoc. 2012;7(3):508-516. https://doi.org/10.1038/nprot.2011.454

14. Mikkonen JJW, Singh SP, Herrala M, Lappalainen R, Myllymaa S, Kullaa AM. Salivary metabolomics in the diagnosis of oral cancer and periodontal diseases. J Periodontal Res. 2016;51(4):431-437. https://doi.org/10.1111/jre.12327

15. Romano F, Meoni G, Manavella V, et al. Effect of non-surgical periodontal therapy on salivary metabolic fingerprint of generalized chronic periodontitis using nuclear magnetic resonance spectroscopy. Arch Oral Biol. 2019;97:208-214. https://doi.org/10.1016/j. archoralbio.2018.10.023

16. Aimetti M, Cacciatore S, Graziano A, Tenori L. Metabonomic analysis of saliva reveals generalized chronic periodontitis signature. Metabolomics. 2012;8(3):465-474. https://doi.org/10.1007/s1130 6-011-0331-2

17. Romano F, Meoni G, Manavella V, et al. Analysis of salivary phenotypes of generalized aggressive and chronic periodontitis through nuclear magnetic resonance-based metabolomics. J Periodontol. 2018;89(12):1452-1460. https://doi.org/10.1002/JPER.18-0097

18. Bostanci N, Belibasakis GN. Gingival crevicular fluid and its immune mediators in the proteomic era. Periodontol 2000. 2018;76(1):6884. https://doi.org/10.1111/prd.12154

19. Taylor JJ, Preshaw PM. Gingival crevicular fluid and saliva. Periodontol 200. 2016;70(1):7-10. https://doi.org/10.1111/ prd.12118

20. Barros SP, Williams R, Offenbacher S, Morelli T. Gingival crevicular fluid as a source of biomarkers for periodontitis. Periodontol 2000. 2016;70(1):53-64. https://doi.org/10.1111/prd.12107

21. Arias-Bujanda N, Regueira-Iglesias A, Balsa-Castro C, Nibali L, Donos N, Tomás I. Accuracy of single molecular biomarkers in gingival crevicular fluid for the diagnosis of periodontitis: A systematic review and meta-analysis. J Clin Periodontol. 2019;46(12):11661182. https://doi.org/10.1111/jcpe.13188

22. Chen X, Yu D. Metabolomics study of oral cancers. Metabolomics. 2019;15:2. https://doi.org/10.1007/s11306-019-1483-8

23. Higgins JPT, Altman DG, Gotzsche PC, et al. The Cochrane Collaboration's tool for assessing risk of bias in randomised trials. BMJ. 2011;343(oct18 2):d5928. https://doi.org/10.1136/bmj.d5928

24. Liberati A, Altman DG, Tetzlaff J, et al. The PRISMA statement for reporting systematic reviews and meta-analyses of studies that evaluate healthcare interventions: explanation and elaboration. BMJ. 2009;339(jul21 1):b2700. https://doi.org/10.1136/bmj.b2700

25. DerSimonian R, Laird N. Meta-analysis in clinical trials. Control Clin Trials. 1986;7(3):177-188. https://doi. org/10.1016/0197-2456(86)90046-2

26. Chen HW, Zhou W, Liao Y, Hu SC, Chen TL, Song ZC. Analysis of metabolic profiles of generalized aggressive periodontitis. J Periodontal Res. 2018;53(5):894-901. https://doi.org/10.1111/ jre.12579

27. Ozeki M, Nozaki T, Aoki J, et al. Metabolomic Analysis of Gingival Crevicular Fluid Using Gas Chromatography/Mass Spectrometry. Mass Spectrom Tokyo. 2016;5(1):A0047. https://doi.org/10.5702/ massspectrometry.A0047

28. Zein Elabdeen HR, Mustafa M, Szklenar M, Rühl R, Ali R, Bolstad Al. Ratio of pro-resolving and pro-inflammatory lipid mediator precursors as potential markers for aggressive periodontitis. Tanowitz HB, ed. PLoS One. 2013;8(8):e70838. https://doi.org/10.1371/journ al.pone. 0070838

29. Pei J, Li F, Xie Y, Liu J, Yu T, Feng X. Microbial and metabolomic analysis of gingival crevicular fluid in general chronic periodontitis patients: lessons for a predictive, preventive, and personalized medical approach. EPMA J. 2020;11(2):197-215. https://doi. org/10.1007/s13167-020-00202-5

30. Hashimura S, Kido J, Matsuda R, et al. A low level of lysophosphatidic acid in human gingival crevicular fluid from patients with periodontitis due to high soluble lysophospholipase activity: Its potential protective role on alveolar bone loss by periodontitis. Biochim Biophys Acta Mol Cell Biol Lipids. 2020;1865(7):158698. https://doi.org/10.1016/j.bbalip.2020.158698

31. Atabay VE, Lutfioğlu M, Avci B, Sakallioglu EE, Aydoğdu A. Obesity and oxidative stress in patients with different periodontal status: a case-control study. J Periodontal Res. 2017;52(1):51-60. https://doi. org/10.1111/jre.12368

32. Ghallab N, Hamdy E, Shaker O. Malondialdehyde, superoxide dismutase and melatonin levels in gingival crevicular fluid of aggressive and chronic periodontitis patients. Aust Dent J. 2016;61(1):53-61. https://doi.org/10.1111/adj.12294

33. Öngöz Dede F, Bozkurt Doğan Ş, Ballı U, Avcı B, Durmuşlar MC. The effect of initial periodontal treatment on plasma, gingival crevicular fluid and salivary levels of 8-hydroxy-deoxyguanosine in obesity. 
Arch Oral Biol. 2016;62:80-85. https://doi.org/10.1016/j.archo ralbio.2015.11.014

34. Öngöz Dede F, Bozkurt Doğan Ş, Balli U, Avci B, Durmuşlar MC, Baratzade T. Glutathione levels in plasma, saliva and gingival crevicular fluid after periodontal therapy in obese and normal weight individuals. J Periodontal Res. 2016;51(6):726-734. https://doi. org/10.1111/jre.12349

35. Hendek MK, Erdemir EO, Kisa U, Ozcan G. Effect of initial periodontal therapy on oxidative stress markers in gingival crevicular fluid, saliva, and serum in smokers and non-smokers with chronic periodontitis. J Periodontol. 2015;86(2):273-282. https://doi. org/10.1902/jop.2014.140338

36. Dede FÖ, Ozden FO, Avcl B. 8-hydroxy-deoxyguanosine levels in gingival crevicular fluid and saliva in patients with chronic periodontitis after initial periodontal treatment. J Periodontol. 2013;84(6):821-828. https://doi.org/10.1902/jop.2012.120195

37. Bathena SP, Huang J, Nunn ME, et al. Quantitative determination of lysophosphatidic acids (LPAs) in human saliva and gingival crevicular fluid (GCF) by LC-MS/MS. J Pharm Biomed Anal. 2011;56(2):402407. https://doi.org/10.1016/j.jpba.2011.05.041

38. Wei D, Zhang X-L, Wang Y-Z, Yang C-X, Chen G. Lipid peroxidation levels, total oxidant status and superoxide dismutase in serum, saliva and gingival crevicular fluid in chronic periodontitis patients before and after periodontal therapy. Aust Dent J. 2010;55(1):7078. https://doi.org/10.1111/j.1834-7819.2009.01123.x

39. Pradeep AR, Kumar MS, Ramachandraprasad MV, Shikha C. Gingival crevicular fluid levels of neopterin in healthy subjects and in patients with different periodontal diseases. J Periodontol. 2007;78(10):1962-1967. https://doi.org/10.1902/ jop.2007.070096

40. Akalin FA, Baltacioğlu E, Alver A, Karabulut E. Lipid peroxidation levels and total oxidant status in serum, saliva and gingival crevicular fluid in patients with chronic periodontitis. J Clin Periodontol. 2007;34(7):558-565. https://doi. org/10.1111/j.1600-051X.2007.01091.x

41. Sun $X$, Mao $Y$, Dai $P$, et al. Mitochondrial dysfunction is involved in the aggravation of periodontitis by diabetes. J Clin Periodontol. 2017;44(5):463-471. https://doi.org/10.1111/jcpe.12711

42. Tonguç MÖ, Öztürk $O$, Sütçü $R$, et al. The impact of smoking status on antioxidant enzyme activity and malondialdehyde levels in chronic periodontitis. J Periodontol. 2011;82(9):1320-1328. https:// doi.org/10.1902/jop.2011.100618

43. Sheikhi M, Bouhafs RK, Hammarström KJ, Jarstrand C. Lipid peroxidation caused by oxygen radicals from Fusobacterium-stimulated neutrophils as a possible model for the emergence of periodontitis. Oral Dis. 2001:7(1):41-46.

44. Rall LC, Roubenoff R, Meydani SN, Han SN, Meydani M. Urinary 8-hydroxy-2'-deoxyguanosine (8-OHdG) as a marker of oxidative stress in rheumatoid arthritis and aging: effect of progressive resistance training. J Nutr Biochem. 2000;11(11-12):581-584. https:// doi.org/10.1016/s0955-2863(00)00123-6

45. Bahar G, Feinmesser R, Shpitzer T, Popovtzer A, Nagler RM Salivary analysis in oral cancer patients: DNA and protein oxidation, reactive nitrogen species, and antioxidant profile. Cancer 2007;109(1):54-59. https://doi.org/10.1002/cncr.22386

46. Su H, Gornitsky M, Velly AM, Yu H, Benarroch M, Schipper HM. Salivary DNA, lipid, and protein oxidation in nonsmokers with periodontal disease. Free Radic Biol Med. 2009;46(7):914-921. https:// doi.org/10.1016/j.freeradbiomed.2009.01.008

47. Łuczaj W, Gęgotek A, Skrzydlewska E. Antioxidants and HNE in redox homeostasis. Free Radic Biol Med. 2017;111:87-101. https:// doi.org/10.1016/j.freeradbiomed.2016.11.033

48. George JJ, Headen KV, Ogunleye AO, et al. Lysophosphatidic Acid signals through specific lysophosphatidic Acid receptor subtypes to control key regenerative responses of human gingival and periodontal ligament fibroblasts. J Periodontol. 2009;80(8):1338 1347. https://doi.org/10.1902/jop.2009.080624

49. Chapple ILC, Brock G, Eftimiadi C, Matthews JB. Glutathione in gingival crevicular fluid and its relation to local antioxidant $\mathrm{ca}-$ pacity in periodontal health and disease. J Clin Pathol - Mol Pathol. 2002;55(6):367-373. https://doi.org/10.1136/mp.55.6.367

50. Gertsman I, Barshop BA. Promises and pitfalls of untargeted metabolomics. J Inherit Metab Dis. 2018;41(3):355-366. https://doi. org/10.1007/s10545-017-0130-7

51. Evans ED, Duvallet C, Chu ND, et al. Predicting human health from biofluid-based metabolomics using machine learning. Sci Rep. 2020;10(1):17635. https://doi.org/10.1038/s41598-020-74823-1

52. Mombelli A. Microbial colonization of the periodontal pocket and its significance for periodontal therapy. Periodontol 2000. 2018;76(1):85-96. https://doi.org/10.1111/prd.12147

53. Baima G, laderosa G, Citterio F, et al. Salivary metabolomics for the diagnosis of periodontal diseases: a systematic review with methodological quality assessment. Metabolomics Off J Metabolomic Soc. 2021;17(1):1. https://doi.org/10.1007/s11306-020-01754-3

54. Wallner-Liebmann S, Tenori L, Mazzoleni A, et al. Individual human metabolic phenotype analyzed by $1 \mathrm{H}$ NMR of saliva samples. J Proteome Res. 2016;15(6):1787-1793. https://doi.org/10.1021/acs. jproteome.5b01060

55. Washio J, Takahashi N. Metabolomic studies of oral biofilm, oral cancer, and beyond. Int J Mol Sci. 2016;17(6): https://doi. org/10.3390/ijms17060870

56. Zhao Y, Hao Z, Zhao C, et al. A novel strategy for large-scale metabolomics study by calibrating gross and systematic errors in gas chromatography-mass spectrometry. Anal Chem. 2016;88(4):22342242. https://doi.org/10.1021/acs.analchem.5b03912

57. Emwas A-H, Roy R, McKay RT, et al. NMR spectroscopy for metabolomics research. Metabolites. 2019;9:7. https://doi.org/10.3390/ metabo9070123

58. Barnes VM, Kennedy AD, Panagakos F, et al. Global metabolomic analysis of human saliva and plasma from healthy and diabetic subjects, with and without periodontal disease. Yilmaz Ö, ed. PLoS One. 2014;9(8):e105181. https://doi.org/10.1371/journal.pone.0105181

59. Bernini P, Bertini I, Luchinat C, Nincheri P, Staderini S, Turano P. Standard operating procedures for pre-analytical handling of blood and urine for metabolomic studies and biobanks. J Biomol NMR. 2011;49(3-4):231-243. https://doi.org/10.1007/s1085 8-011-9489-1

60. Emwas A-H, Roy R, McKay RT, et al. Recommendations and standardization of biomarker quantification using NMR-Based metabolomics with particular focus on urinary analysis. J Proteome Res. 2016;15(2):360-373. https://doi.org/10.1021/acs.jprot eome.5b00885

61. Emwas A-H, Luchinat $\mathrm{C}$, Turano $\mathrm{P}$, et al. Standardizing the experimental conditions for using urine in NMR-based metabolomic studies with a particular focus on diagnostic studies: a review. Metabolomics. 2015;11(4):872-894. https://doi.org/10.1007/s11306-014-0746-7

62. Rasmussen L, Hänström L, Lerner UH. Characterization of bone resorbing activity in gingival crevicular fluid from patients with periodontitis. J Clin Periodontol. 2000;27(1):41-52. https://doi. org/10.1034/j.1600-051x.2000.027001041.x

63. Silva-Boghossian CM,Colombo AP, Tanaka M, Rayo C,XiaoY, Siqueira WL. Quantitative proteomic analysis of gingival crevicular fluid in different periodontal conditions. PLoS One. 2013;8(10):e75898. https://doi.org/10.1371/journal.pone.0075898

64. Teles R, Moss K, Preisser JS, et al. Patterns of periodontal disease progression based on linear mixed models of clinical attachment loss. J Clin Periodontol. 2018;45(1):15-25. https://doi.org/10.1111/ jcpe.12827

65. Aimetti M, Fratini A, Manavella $\mathrm{V}$, et al. Pocket resolution in regenerative treatment of intrabony defects with papilla preservation 
techniques: a systematic review and meta-analysis of randomized clinical trials. J Clin Periodontol. 2021. Jan 21. https://doi. org/10.1111/jcpe.13428. Epub ahead of print.

\section{SUPPORTING INFORMATION}

Additional supporting information may be found online in the Supporting Information section.
How to cite this article: Baima G, Corana M, laderosa G, et al. Metabolomics of gingival crevicular fluid to identify biomarkers for periodontitis: A systematic review with meta-analysis. J Periodont Res. 2021;00:1-13. https://doi. org/10.1111/jre.12872 